\title{
Somatic DNA copy number alterations and their potential clinical utility for predicting lethal prostate cancer
}

\author{
Wennuan $\mathrm{Liu}^{1,2}$, Li Wang ${ }^{1,2}$ and Jianfeng $\mathrm{Xu}^{1,2,3}$ \\ Asian Journal of Andrology (2013) 15, 586-587; doi:10.1038/aja.2013.82; published online 1 July 2013
}

\begin{abstract}
urrent clinicopathologic indicators $\checkmark$ are insufficient to distinguish the small percentage of aggressive prostate cancer (PCa) from the vast majority of indolent disease at diagnosis, leading to overtreatment of PCa. A recent study reported and confirmed a strong association of PCaspecific mortality with somatic DNA copy number alterations (CNAs) in primary prostate tumors. If a sensitive, robust and lowcost method can be developed to measure these CNAs in biopsy specimens, it may help to stratify patients for more appropriate management at the time of diagnosis; aggressive treatment or active surveillance for patients with or without these CNAs, respectively.
\end{abstract}

A study on the association of somatic DNA copy number alterations (CNAs) in prostate tumors with lethal prostate cancer (PCa) by our collaborative group of Wake Forest University School of Medicine, the Brady Urological Institute of Johns Hopkins Medical Institutions, and the Karolinska Institute of Sweden was recently published in Cancer. ${ }^{1}$ Shortly after, two abstracts on the association of RNA expression of two different panels of genes with progression of PCa were presented by investigators from Mayo Clinic and University of California at San Francisco, respectively, in the 2013 American Urology Association Annual meeting. ${ }^{2,3}$ These studies all focused on the prognosis of PCa and received great attention in the scientific and clinical communities as well as in public, as evidenced by the news coverage. The excitement surrounding these studies is not surprising because distinguishing aggressive from

${ }^{1}$ Center for Cancer Genomics, Winston-Salem, NC 27157, USA; ${ }^{2}$ Center for Genomics and Personalized Medicine Research, Wake Forest University School of Medicine, Winston-Salem, NC 27157, USA and ${ }^{3}$ Fudan Institute of Urology, Huashan Hospital, Fudan University, Shanghai 200040, China

Correspondence: Dr JF Xu (jxu@wfubmc.edu) indolent forms of PCa is probably the most important clinical task in managing and treating this heterogeneous disease. Most prostate tumors grow so slowly that they may never threaten a man's life; however, $\sim 15 \%$ are lethal if not treated appropriately. Currently used clinical predictors such as Gleason score and tumor stages are useful but insufficient to discriminate between the two types of PCa at the time of diagnosis. This inability leads to unnecessary and intensive treatment of many PCa patients, reducing their quality of life, and incurring financial burden to patients, families and society. Novel biomarkers are urgently needed to better identify aggressive PCa at the time of diagnosis for selection of treatment strategy; a choice of active surveillance for indolent $\mathrm{PCa}$ or intensive treatment for aggressive PCa.

In this perspective piece, we highlight the key points of our Cancer paper on the association of CNAs with lethal $\mathrm{PCa},{ }^{1}$ discuss their advantages as markers for lethal $\mathrm{PCa}$ and describe the challenges for translating the genomic findings to clinics.

\section{DISCOVERY OF 7 CNAs ASSOCIATED WITH LETHAL PCa}

Using Affymetrix 6.0 SNP array to analyze tumor and matched normal DNA from 125 prostatectomy patients treated at Johns Hopkins Hospital and the algorithm of Genomic Identification of Significant Targets in Cancer, we uncovered seven regions in the tumor genome that were significantly associated with lethal PCa. These include CNAs of MYC at 8q24.21, SERPINB5 at 18q21.33, TPD52 at $8 \mathrm{q} 21.13$, USP10 at $16 \mathrm{q} 24.1$, PTEN at $10 \mathrm{q} 23.31, T P 53$ at $17 \mathrm{p} 13.1$ and $A D A R$ at 1q21.3. Note that these CNAs not only play a role in the development of $\mathrm{PCa}$ (comparing tumor versus normal), but more importantly they may play a role in progression of $\mathrm{PCa}$ (comparing lethal PCa patients versus patients that are still alive)

\section{PTEN AND MYC ARE INDEPENDENT} PREDICTORS OF LETHAL PCa

When adjusting for other known predictors of lethal PCa such as Gleason score and pathological stage using a multivariate logistic regression analysis, we found that two of these seven CNAs (PTEN deletion and $M Y C$ amplification) remained statistically significant. These results suggest that PTEN deletion and MYC amplification conferred additional prognostic value; therefore, they have potential to improve current clinical practice to distinguish lethal from indolent PCa.

\section{COMBINED EFFECT OF PTEN LOSS AND} MYC GAIN ON MORTALITY OF PCa

Compared with patients without alterations at either PTEN or MYC, patients with both alterations had 53-fold higher risk for dying from the disease. It is important to note that although several prior studies had implicated PTEN loss in PCa metastasis, ${ }^{4,5}$ PTEN loss in death from hormone refractory $\mathrm{PCa},{ }^{6}$ and the loss of PTEN and gain of MYC in metastatic disease, ${ }^{7}$ our study was the first to demonstrate a stronger joint effect of PTEN and $M Y C$ on PCa-specific mortality.

\section{CONFIRMATION OF CNAS AT PTENIMYC} WITH PCa-SPECIFIC MORTALITY

An important aspect of the study was the further confirmation of the joint effect in 333 tumors from three additional distinct patient cohorts, including 103 patients who underwent prostatectomy at the Karolinska University Hospital in Sweden, 216 patients treated at Memorial Sloan Kettering Cancer Center and 14 autopsy patients from Johns Hopkins Hospital. For biomarker studies of whole genome analysis, independent 
confirmation is necessary to reduce the chance of false positive findings due to multipletesting issues in the discovery stage.

\section{ADVANTAGE OF SOMATIC CNAs AS A MARKER FOR LETHAL PCa}

Acquired DNA alterations in cancer cells accumulate and are preserved over time for as long as 10 years. ${ }^{8,9}$ Natural selection of somatic cells with DNA alterations that confer increased proliferation, decreased apoptosis or ability of invasion is the genetic basis of tumor development and clonal phenomena. In fact, monoclonal origins of lethal metastatic PCa have been observed. ${ }^{10}$ Because CNAs precede phenotypic presentations or histological changes, the discovery of CNAs associated with $\mathrm{PCa}$-specific death may help identify a subset of patients with particularly aggressive cancers at an early stage, for targeted aggressive treatment.

\section{CHALLENGES AND OPPORTUNITIES FOR CLINICAL TRANSLATION}

In order to utilize these genomic findings for determining treatment options, these CNAs will most likely need to be detected in biopsy specimens at time of diagnosis. This poses significant challenges due to the multi-foci nature of PCa (heterogeneity), small amount of tumor cells in each cancer foci (insufficient tumor DNA and contamination of normal
DNA), and the fragmentation of DNA molecules in formalin-fixed, paraffin-embedded biopsy specimens. Therefore, detection methods that are sensitive to small amounts of tumor DNA, robust to fragmented DNA, high-throughput for the measurement of all possible CNAs at the same time, and low cost, are needed in order to assay each needle biopsy core to identify signatures of CNAs that are associated with lethal $\mathrm{PCa}$. Fortunately, quantitative PCR and multiplex ligation-dependent probe amplification may meet these criteria. However, studies are needed to demonstrate the capability of these methods to detect these CNAs in individual biopsy cores, and their predictive performance for the detection of lethal PCa.

With the discovery of CNAs associated with lethal PCa, there is hope that new diagnostic models can provide a more accurate prognosis at the time of $\mathrm{PCa}$ diagnosis. This in turn can inform treatment that is tailored to each patient, whereby patients with particular DNA signatures of lethal PCa can be targeted for more aggressive treatment, while patients without these CNAs may opt for active surveillance.

1 Liu W, Xie CC, Thomas CY, Kim ST, Lindberg J et al. Genetic markers associated with early cancer-specific mortality following prostatectomy. Cancer; e-pub ahead of print 22 April 2013; doi:10.1002/ cncr.27954.

2 Cooperberg M, Simko J, Falzarano S, Maddala T, Chan J et al. Development and validation of the biopsy-based genomic prostate score (GPS) as a predictor of high grade or extracapsular prostate cancer to improve patient selection for active surveillance. Proc Am Urol Assoc 2013 Annu Meet 2013; 2013: Abstract 2131.

3 Karnes RJ, Ghadessi M, Bergstralh EJ, Erho N, Crisan A et al. Validation of a genomic classifier that predicts metastatic disease progression in men with high risk pathological features post-prostatectomy. Proc Am Urol Assoc 2013 Annu Meet 2013; 2013: Abstract 2130.

4 Yoshimoto M, Cunha IW, Coudry RA, Fonseca FP, Torres $\mathrm{CH}$ et al. Fish analysis of 107 prostate cancers shows that pten genomic deletion is associated with poor clinical outcome. Br J Cancer 2007; 97: 678-85.

5 Ding Z, Wu CJ, Chu GC, Xiao Y, Ho D et al. Smad4 dependent barrier constrains prostate cancer growth and metastatic progression. Nature 2011;470: 269 73.

6 Sircar K, Yoshimoto M, Monzon FA, Koumakpayi IH, Katz RL et al. Pten genomic deletion is associated with p-AKT and AR signalling in poorer outcome, hormone refractory prostate cancer. J Pathol 2009; 218: 505-13.

7 Lapointe J, LiC, Giacomini CP, Salari K, Huang S etal. Genomic profiling reveals alternative genetic pathways of prostate tumorigenesis. Cancer Res 2007; 67: 8504-10.

8 Luebeck EG. Cancer: Genomic evolution of metastasis. Nature 2010; 467: 1053-5.

9 Baca SC, Prandi D, Lawrence MS, Mosquera JM, Romanel $\mathrm{A}$ et al. Punctuated evolution of prostate cancer genomes. Cell 2013; 153: 666-77.

10 Liu W, Laitinen S, Khan S, Vihinen M, Kowalski J et al. Copy number analysis indicates monoclonal origin of lethal metastatic prostate cancer. Nat Med 2009; 15 $559-65$. 